\title{
UMA ARQUITETURA FLEXÍVEL PARA PROMOVER A INTEGRAÇÃO ENTRE O MOODLE E SITES DE REDES SOCIAIS
}

\author{
Title: A Flexible Architecture to Promote the Integration between Moodle and Social \\ Network Sites
}

\author{
Lucas M. Braz \\ UNISINOS - Av. Unisinos, 950 - B \\ CEP 93.022-000 - São Leopoldo/RS \\ lmonteirobraz@gmail.com \\ Sérgio Crespo C. S. Pinto \\ UNISINOS - Av. Unisinos, 950 - B \\ CEP 93.022-000 - São Leopoldo/RS \\ crespo@unisinos.br
}

\author{
Tássia Serrão \\ UNISINOS - Av. Unisinos, 950 - B \\ CEP 93.022-000 - São Leopoldo/RS \\ tassiaserrao@gmail.com \\ Gisela Clunie \\ Univeridad Tecnológica de Panamá (UTP) \\ gisela.clunie@utp.ac.pa
}

Resumo Sites de redes sociais possibilitam que internautas se comuniquem, interajam, colaborem e expressem suas ideias. Também conhecidos por SNSs, eles exercem uma impressionante atração sobre seus usuários, os quais passam diversas horas por dia nesse tipo de sites. Este artigo defende a ideia de que a popularidade e as diversas ferramentas de socialização que esses sites oferecem podem ser utilizadas em prol da educação, como forma de aumentar o interesse e o engajamento dos estudantes no processo de ensino/aprendizagem. Para incorporar os SNSs ao processo, este artigo apresenta uma arquitetura flexível para integrar o ambiente Moodle a diferentes sites de redes sociais. Além disso, essa arquitetura foi implementada de forma a realizar a integração do Moodle com Facebook e Twitter. A partir dessa integração, acreditamos que o professor passa a dispor de uma nova forma de comunicação com seus alunos, aumentando as chances de interação entre eles, além de, possivelmente, incentivar os estudantes a serem mais ativos e a utilizarem mais o Moodle.

Palavras-Chave: Arquitetura flexível, Sites de redes sociais, Moodle

\begin{abstract}
Social networking sites make it possible for Internet users to communicating, sharing, cooperating and expressing their ideas. Also known as SNSs, theywield impressive attraction within its users, who spend several daily hours in this kind of sites. This paper advocates that the popularity and social tools these sites provide can be used in behalf of education, as a mean to increase student's interest and commitment for the teaching/learning process. In order to incorporate SNSS into the process, this article presents a flexible architecture to integrate Moodle and distinct social networking sites. Besides, these architecture has been implemented to provide integration between Moodle and Facebook and Twitter. This way, we believe that teachers are provided with a new way of communicating with their students, increasing the odds of interaction among them and, possibly, students get more active and start using Moodle more frequently.
\end{abstract}

Keywords: Flexible architecture, Social networking sites, Moodle 


\section{Introdução}

Sites de redes sociais, como Facebook, Twitter, LinkedIn e MySpace se transformaram em um fenômeno que atrai milhares de novos usuários todos os dias. Eles possibilitam que internautas se comuniquem, interajam, colaborem, expressem suas ideias, suas opiniões e criem/liderem discussões a respeito dos mais variados temas. Também conhecidos por SNSs (do termo em inglês Social Networking Sites), os sites de redes sociais exercem uma impressionante atração especialmente sobre os adolescentes (faixa etária entre 14 e 17 anos) e os chamados jovens adultos (adultos com menos de 30 anos), os quais passam diversas horas por dia nesse tipo de site postando fotos, vídeos, comentários; ou seja, socializando. Contudo, apesar da sua popularidade, os SNS são vistos com bastante desconfiança por parte de muitos pais e professores preocupados com os possíveis malefícios que o uso desses sites pode trazer aos jovens. Normalmente, essas preocupações estão associadas à privacidade do conteúdo publicado, à possibilidade de que os jovens sofram algum tipo de assédio sexual ou ainda que os sites possam distrair os alunos, afastando-os dos estudos. Como consequência, diversas escolas optaram por bloquear o acesso dos estudantes aos SNS [1]. Nesse sentido, a discussão na comunidade educacional é bastante recente e ainda não existem trabalhos conclusivos sobre o impacto que os SNS têm sobre os estudantes. Entretanto, diversos pesquisadores acreditam que o uso de sites de redes sociais pode ser benéfico ao aprendizado. Ahn [2] afirma que talvez professores possam utilizar SNS para engajar seus estudantes, desenvolver relações mais estreitas e incentivar comportamentos de aprendizagem. Ito et al. [3] defendem que os estudantes aprendem de novas maneiras quando utilizam mídias sociais e que os educadores deveriam adotá-las. Ractham e Firpo [4] sugerem que os SNS possuem grande potencial para expandir o ensino além das salas de aula e que podem ser usados efetivamente para desenvolver uma cultura de aprendizagem. Segundo Jiang e Tang [5], deveria existir um novo modelo de educação baseado em redes sociais para permitir que as pessoas aprendam enquanto elas socializam. Diversos pesquisadores, como Hodgins [6] e McInerney [7], consideram que o conhecimento é construído socialmente através de processos educacionais facilitados por cooperação, colaboração e interações sociais [8] e que o aprendizado deve ocorrer em um contexto socialmente aberto que ofereça diversas formas de interação, como discussões, comentários, ou criação conjunta de recursos educacionais [9].

Nesse sentido, acreditamos que os sites de redes sociais possibilitam que os alunos explorem as características sociais do aprendizado, à medida que oferecem diversas formas de interação, facilitando com que estudantes e professores se comuniquem e colaborem uns com os outros. Embora as preocupações relacionadas a esses sites sejam plausíveis, são também contornáveis. Por exemplo, os principais SNS oferecem controles de privacidade. Além disso, pesquisas comprovam que o uso da tecnologia por si só não afeta o aprendizado dos estudantes [10, 11], mas os comportamentos que ocorrem dentro desses sistemas é que pode gerar resultados positivos ou não. Portanto, em vez de proibir o uso dos SNS, os professores poderiam explorar as novas possibilidades que essas ferramentas oferecem, orientando os alunos a usá-las de forma adequada para produzir resultados educacionais positivos. socializam. Segundo Rivera [12], o potencial dos sites de redes sociais é evidente e que, portanto, a questão é utilizar estrategicamente esse potencial para atingir outros objetivos que não o simples entretenimento.

Este trabalho propõe uma arquitetura flexível para promover a integração entre o LMS (Learning Management System) Moodle e diferentes sites de redes sociais, como por exemplo o Facebook. Essa arquitetura visa: conduzir informações geradas no Moodle para os SNS, informando aos alunos sobre o que acontece no curso (e.g. alertar que o professor criou uma nova atividade); permitir que os estudantes, a partir dos SNS, interajam com os recursos do Moodle, como por exemplo responder a um questionário; e ser flexível de forma que diversos SNS possam ser utilizados. Além disso, este trabalho apresenta uma implementação dessa arquitetura para promover a integração do Moodle com os SNS Facebook e Twitter, como forma de evidenciar a flexibilidade da arquitetura, a qual pode ser estendida para o uso de outros SNS. A partir desta integração Moodle / SNS, o professor passa a dispor de uma nova forma de se comunicar com seus alunos. As interações, antes restritas ao ambiente do Moodle, podem ocorrer também em uma ferramenta que sabidamente está incorporada ao cotidiano dos estudantes. Portanto, passa a existir um novo canal de comunicação entre professores e alunos, aumentando as chances de que as informações cheguem efetivamente aos estudantes, podendo ainda serem compartilhadas, comentadas e discutidas. Além disso, o uso dos SNS pode motivar os alunos a serem mais ativos no processo e, inclusive, incentivá-los a utilizarem mais o Moodle. Este trabalho apresenta uma arquitetura para promover a integração, de forma flexível, entre diferentes sites de redes sociais e o sistema de gerenciamento de aprendizagem Moodle. Esta arquitetura possibilita a construção de uma ponte entre professores e alunos para facilitar a comunicação entre eles, de forma que as informações cheguem aos alunos onde eles estão (i.e. sites de redes sociais). A arquitetura proposta gerencia e conduz as informações em duas vias: desde os LMS até os sites de redes sociais, bem como o 
caminho inverso. Assim, por um lado, os professores podem comunicar aos alunos sobre provas, entrega de trabalhos, exercícios, ou mesmo eventos que ocorram em suas instituições como palestras e conferências, além de poderem estimulá-los a discutir sobre assuntos relevantes à disciplina; por outro lado, os alunos têm a possibilidade de usar um ambiente que eles acessam frequentemente para ficarem bem informados sobre o que está acontecendo em seus cursos, liderarem e/ou se engajarem em discussões importantes, compartilharem recursos (arquivos, fotos, vídeos) e colaborarem uns com os outros.

\section{Fundamentação Teórica}

Esta seção apresenta a fundamentação teórica referente ao foco deste trabalho, abordando as tecnologias utilizadas e os conceitos correlacionados.

\subsection{Redes Sociais}

O termo 'redes sociais' tem recebido bastante atenção recentemente, sendo foco de reportagens em revistas, jornais, programas de TV e até mesmo filmes de sucesso. Normalmente associado a uma quantidade exorbitante (e crescente) de usuários e lucros milionários, esse termo indubitavelmente está em voga e atrai interesse de pesquisadores e empresas.

O conceito de redes sociais não é novo, já existia antes mesmo do surgimento da Web e tem sido aplicado em diversas áreas da sociologia para a compreensão de diferentes eventos sociais, políticos, econômicos, afetivos, entre outros [13]. Como definido por Garton et al. [14] e Serrat [15], uma rede social é um grupo de pessoas que estão conectadas por um conjunto de relacionamentos, como amizade, trabalho ou interesses comuns. Assim, uma rede social tem como foco os relacionamentos e qualquer pessoa que possui família, amigos, colegas, isto é, que se relacione, faz parte de uma ou (provavelmente) várias redes sociais. Entretanto, a popularização do termo ocorreu devido ao advento dos chamados sites de redes sociais, como Friendster, Facebook, Flicker, LinkedIn, Orkut ou MySpace, os quais tem um poder surpreendente de atrair e engajar milhões de usuários ao redor do mundo. Para citar um exemplo, o Facebook possui mais de 937 milhões de usuários em todo o globo [16]. Os sites de redes sociais (também chamados SNSs) são espaços na Internet onde comunidades ou grupos de pessoas são criados por meio de ligações sociais, nos quais os participantes compartilham atividades ou interesses similares e podem se expressar e se comunicar uns com os outros $[12,17]$. Em geral, esses sites possuem um mesmo conjunto básico de funcionalidades. Eles permitem que os usuários: construam um perfil público, onde é possível adicionar uma foto e descrever detalhes pessoais; articulem uma lista de outros usuários com os quais eles compartilham uma conexão; visualizem suas listas de cone- xões e a de outros usuários do sistema; escrevam comentários; criem e participem de discussões em fóruns; e realizem upload de diferentes mídias, como fotos ou vídeos $[18,19]$. O que diferencia os sites de redes sociais de qualquer outra forma de comunicação mediada por computador, de acordo com Boyd e Ellison [18], é o fato de que eles tornam visíveis as redes sociais e permitem que os usuários articulem e desenvolvam suas redes. Essa é uma característica muito importante, pois uma rede social é tão valiosa quanto as pessoas e conexões que se pode enxergar.

Para exemplificar, consideremos o cenário ilustrado na Figura 1: uma pessoa chamada Joana, que é amiga de José, o qual conhece Maria, que é amiga de Carlos, o qual poderia oferecer um emprego para Joana. No mundo real os relacionamentos estão ocultos. Joana dificilmente saberia como contactar Carlos para conseguir o emprego. Os SNSs tornam as redes visíveis possibilitando que as oportunidades que elas oferecem sejam aproveitadas.

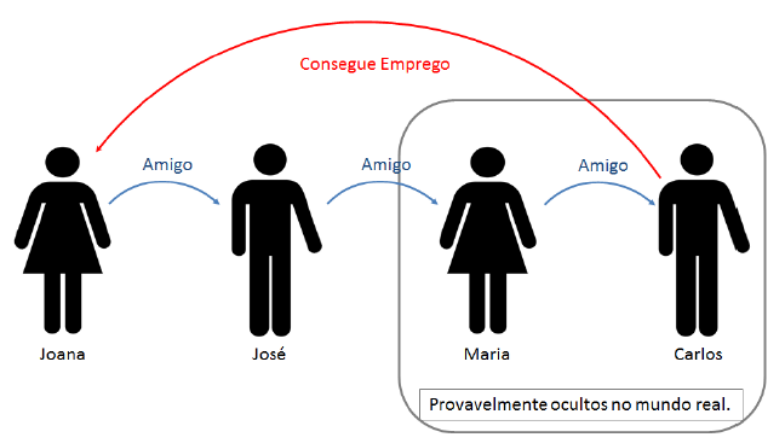

Figura 1 - Potencial dos sites de redes sociais.

Segundo Rivera [12], o potencial dos sites de redes sociais é evidente. Portanto, a questão que surge é: como utilizar estrategicamente esse potencial para atingir outros objetivos que não o simples entretenimento?

\subsection{Redes Sociais na Educação}

Os sites de redes sociais são uma ótima ferramenta para engajar pessoas, provendo facilidade de comunicação de forma que os usuários podem interagir de diversas maneiras e possivelmente realizarem algum tipo de colaboração. Essas ferramentas trazem várias oportunidades para o aprendizado e podem auxiliar o professor na tarefa de estimular os alunos a serem mais participativos no processo de ensino-aprendizagem. As possibilidades que as redes sociais podem trazer à educação tem atraído diversos pesquisadores a investigar se o uso desse tipo de ferramenta é de fato benéfico. Ractham e Firpo [4] conduziram um experimento onde tentaram utilizar o Facebook em um curso universitário como um recurso educacional para os estudantes compartilharem seus conhecimentos e experiências. $\mathrm{O}$ experimento durou 5 meses e 
envolveu 69 alunos. Eles concluíram que os SNSs podem ser usados efetivamente para desenvolver uma cultura de aprendizagem e que esses sites tem grande potencial para expandir o ensino além das salas de aula. De forma semelhante, Lockyer e Patterson [20] realizaram um estudo de caso onde eles tentaram utilizar o Flicker como ferramenta de apoio ao ensino. O estudo teve duração de 13 semanas, com a participação de 12 estudantes. A partir dessa experiência eles afirmaram que incorporar o SNS a um ambiente de educação formal trouxe resultados positivos aos participantes, porém ressaltam o cuidado que se deve ter com relação à privacidade quando se utiliza esse tipo de site. Li e Liu [21] realizaram um estudo semelhante utilizando o SNS Xiaonei e também chegaram a conclusão de que o uso de sites de redes sociais é relevante e benéfico para o ensino. Jiang e Tang [5] afirmam que deveria existir um novo modelo de educação baseado em redes sociais para permitir que as pessoas aprendam enquanto elas socializam. Maglajlic et al [22] e Cain [23] são outros exemplos de pesquisas que defendem a utilização dos SNSs como ferramentas de apoio ao processo de aprendizagem.

\subsection{Ontologias}

Gruber [24] descreve uma ontologia como uma representação formal e explícita de um conhecimento compartilhado. Ou seja, uma ontologia torna explícito o conhecimento embutido em um conjunto de dados e apresenta esse conhecimento de forma estruturada (formal), o que possibilita a construção de entidades de software capazes de processar e extrair significado desses dados. Para a construção de ontologias na Web, pode-se utilizar a linguagem OWL (Ontology Web Language). Essa linguagem permite um alto grau de expressividade e inferência implícita. A OWL foi construída baseada em RDF, e portanto herda todas as suas qualidades e características, além de ser uma recomendação da W3C [25]. A OWL foi dividida em três sublinguagens, ou dialetos, de acordo com as suas capacidades de expressividade e garantia de computabilidade:

- OWL Lite: é a sub-linguagem mais simples da OWL. Possui pouca expressividade se comparada às demais sub-linguagens e deve ser utilizada para a definição de hierarquias e restrições simples;

- OWL DL: essa sub-linguagem possui o máximo de expressividade e ao mesmo tempo garante decidibilidade. Ela permite a verificação de satisfatibilidade de conceitos, classificação de hierarquias e inferência de tipos. Para garantir a decidibilidade computacional, a OWL DL impõe algumas restrições, por exemplo uma classe não pode ser definida como uma instância de outra classe;
- OWL Full: essa sub-linguagem possui o máximo de expressividade mas sem as restrições impostas pela OWL DL. No entanto, ela não oferece garantias computacionais. Portanto, essa linguagem deve ser utilizada quando a expressividade do conhecimento for mais importante que a garantia de computabilidade.

\subsection{Agentes de Software}

A tecnologia de agentes de software surgiu como um novo paradigma no desenvolvimento de sistemas de software complexos e vem sendo utilizada nas mais variadas aplicações, como gerenciamento de rede, sistemas de diagnóstico, controle de processos e logística [26]. Existem várias definições de agentes de software na literatura. De acordo com Russell e Norvig [27], um agente é uma entidade que percebe seu ambiente através de sensores e atua nele por meio de atuadores. Eles exemplificam sua definição diferenciando agentes humanos e agentes de software da seguinte forma: os humanos possuem olhos, ouvidos e outros órgãos como sensores, e mãos, pés, boca e outras partes do corpo como atuadores; já os agentes de software possuem bits decodificados como seus sensores e atuadores. De forma semelhante, Wooldridge [28] considera que um agente de software é um sistema computacional encapsulado que está situado em um determinado ambiente e é capaz de ação flexível autônoma neste ambiente, a fim de alcançar seus objetivos de projeto. Complementando essas definições, Shiang, Kulathuramaiyer e Loke [29] afirmam que os agentes são capazes de planejamento, comunicação, cooperação, coordenação e negociação com outros agentes e usuários, objetivando realizar determinada tarefa ou objetivo. Dentre as características dos agentes de software, podemos destacar:

- Autonomia - habilidade do software de agir independentemente sem intervenção direta humana ou de outros agentes;

- Proatividade - os agentes podem exibir proatividade, comportamento dirigido a objetivos e tomar a iniciativa quando necessário;

- Sociabilidade - habilidade de participar de muitos relacionamentos, interagindo com outros agentes ao mesmo tempo ou em tempos distintos.

Uma outra característica importante dos agentes de software é a flexibilidade. Um agente pode se adequar ao seu ambiente e exibir comportamentos distintos à medida que evolui e aprende. A partir de uma visão arquitetural, os agentes são partições de software que podem ter seu funcionamento modificado de forma isolada, para atender aos mais variados requisitos, isto é, é possível alterar um determinado agente sem impactar outras partes do sistema, facilitando a sua manutenção e evolução. 


\subsection{JADE}

JADE (Java Agent Development Framework) [26] é um framework de código aberto para o desenvolvimento de sistemas multi-agente, implementado em JAVA e que segue as especificações da FIPA (Foundation for Intelligent Physical Agents) [30]. O JADE também suporta a criação de agentes distribuídos entre várias

máquinas (as quais não necessariamente possuem o mesmo sistema operacional) e provê todo o mecanismo de comunicação entre eles. O JADE oferece suporte ao uso de ontologias como linguagem de comunicação entre os agentes de software [26]. Ele possibilita a criação de ontologias a partir de classes Java, onde cada classe representa um conceito e seus atributos constituem as propriedades dos conceitos. Nesse sentido, cada instância de uma determinada classe constitui uma instância do conceito que ela representa. As ontologias são fundamentais na comunicação entre os agentes de software, pois cada agente possui um determinado conhecimento sobre o mundo e quando eles se comunicam, é necessário que eles se entendam. As ontologias oferecem um meio de fazer com que as informações que um determinado agente envia sejam compreendidas corretamente pelos destinatários. No JADE, as mensagens trocadas entre os agentes são em formato de texto, entretanto, há um mecanismo que converte automaticamente a ontologia em texto, no remetente, e converte de texto em ontologia, no destinatário. Outra importante funcionalidade oferecida pelo JADE é o chamado JadeGateway [31]. Ele simula a existência de um agente em uma aplicação Java comum (sem agentes de software) e possibilita a comunicação entre sistemas criados com paradigmas diferentes. Isso é particularmente útil para a integrar uma aplicação construída baseada em agentes a uma aplicação Web, como ocorre na arquitetura desenvolvida neste trabalho.

\section{TRABALHOS RELACIONADOS}

O objetivo desta seção é apresentar uma breve descrição de alguns trabalhos que demonstram certa similaridade com este. De maneira geral, esses trabalhos procuram incorporar as funcionalidades dos sites de redes sociais aos sistemas de gestão de aprendizagem com o intuito de tornar esses sistemas mais sociais e, a partir dessa integração, atrair os estudantes a utilizarem cada vez mais os LMSs. No entanto, esta proposta de dissertação objetiva também o caminho contrário: levar as informações do LMS aos sites de redes sociais, de forma que o aluno possa aprender utilizando uma ferramenta que lhe é familiar e agradável, e que pode the prover diversas formas de interação, tanto com o professor quanto com outros estudantes.

\subsection{Módulos do Moodle}

O Moodle conta com uma grande comunidade ativa de desenvolvedores que estão constantemente criando novos módulos que podem ser incorporados a este LMS. Alguns desses módulos propõem a integração do Moodle com algum serviço de redes sociais. Fulton [32] apresenta um módulo para integrar o Moodle ao Facebook, o qual possibilita: (i) associar uma conta do Facebook à conta do Moodle; (ii) utilizar a conta do Facebook para autenticação no Moodle; (iii) permite que os professores adicionem uma atividade ao curso, denominada Facebook Live Stream Box, a qual permite que os usuários compartilhem comentários em tempo real utilizando a interface do SNS (vide Figura 2).

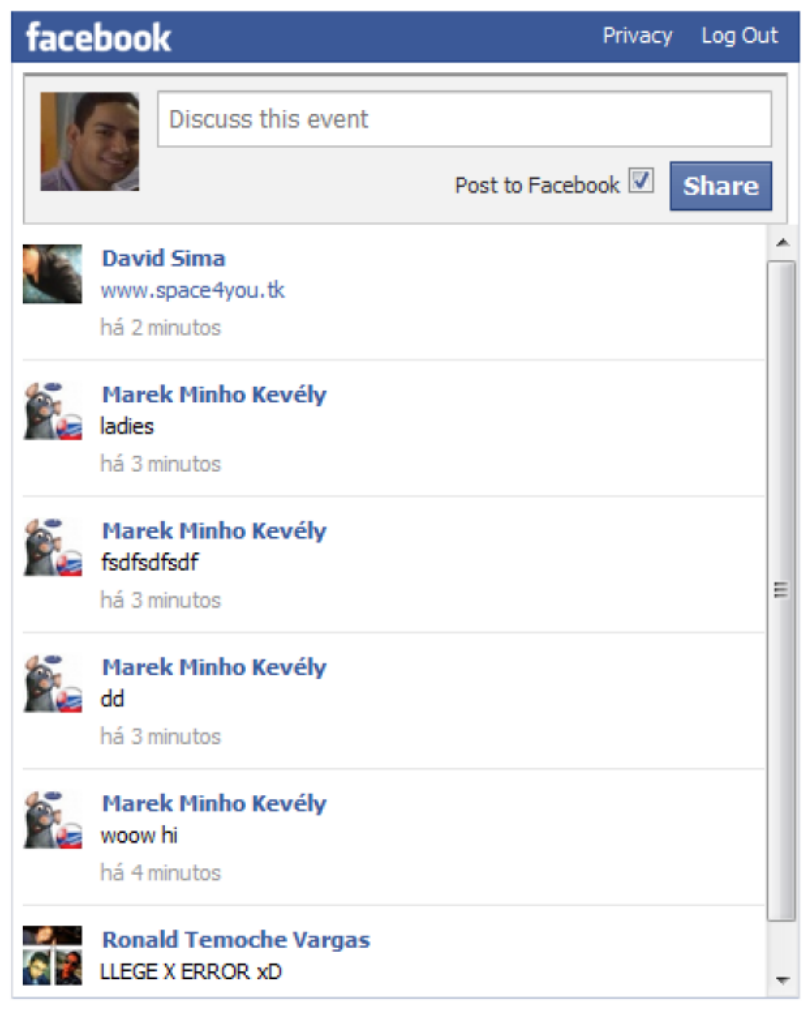

Figura 2 - Exemplo da Facebook Live Stream Box

Ho [33] propõe a integração entre o sistema de gestão de aprendizagem Moodle e o site de redes sociais Facebook com o objetivo de prover o LMS com as capacidades de compartilhamento de recursos do SNS. A integração é proposta através de dois métodos diferentes: (i) desenvolvimento de um módulo, denominado no Moodle como 'bloco', para servir como interface gráfica para o SNS; e (ii) implementação de uma 'atividade' no Moodle que pode ser utilizada como uma das diferentes ações disponíveis em um curso. 


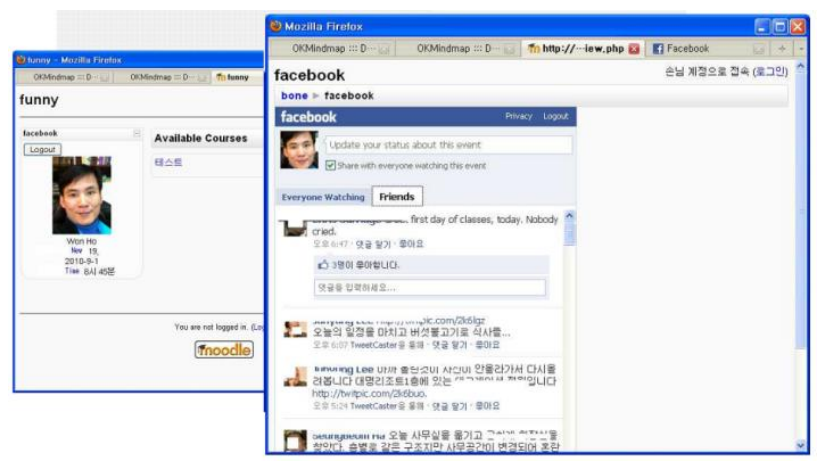

Figura 3 - Módulo permite que usuários leiam informações do Facebook no Moodle (Fonte Ho [33]).

A abordagem utilizada nesse artigo consiste em recuperar informações do SNS e apresenta-las dentro do ambiente Moodle. O 'bloco' desenvolvido permite que os usuários visualizem o seu mural do Facebook e possam atualizá-lo sem a necessidade de sair do site do Moodle, como mostrado na Figura 3. Já a 'atividade' descrita foi implementada como um plugin do Moodle para permitir que os estudantes construam um mapa de conceitos de maneira colaborativa, onde eles podem inserir texto e imagens como nodos, bem como importar dados do $\mathrm{Fa}$ cebook e Twitter (vide Figura 4).

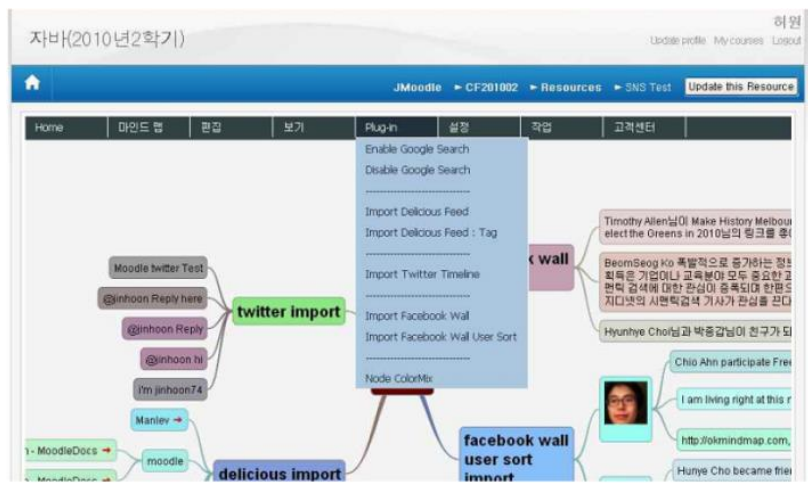

Figura 4 - Mapa de conceitos construido com dados do Facebook e Twitter (Fonte Ho [33])

\subsection{TOPYX}

TOPYX é uma solução de LMS social, implementada na forma de software como serviço (SaaS), que combina os recursos de um LMS tradicional com as ferramentas de redes sociais como Facebook, LinkedIn e Twitter, com o intuito de prover aos usuários uma experiência de aprendizado colaborativo e atraente [34]. Este software foi desenvolvido como uma extensão do LMS Moodle para prover interface gráfica customizada e integração com os sites de redes sociais. Contudo, a empresa responsável não divulga publicamente a maioria das suas funcionalidades, limitando-se a apresentar uma lista de clientes importantes e alguns prêmios conquistados. $\mathrm{O}$ uso desta solução tem o custo de quinze mil dólares anuais, na maioria dos casos [34].

\subsection{Edu 2.0}

Ivanova e Popova [35] apresentam o Edu 2.0, um sistema de gestão de aprendizagem construído com o objetivo de potencializar as interações entre alunos e professores por meio de um conjunto de variadas ferramentas sociais daWeb 2.0.

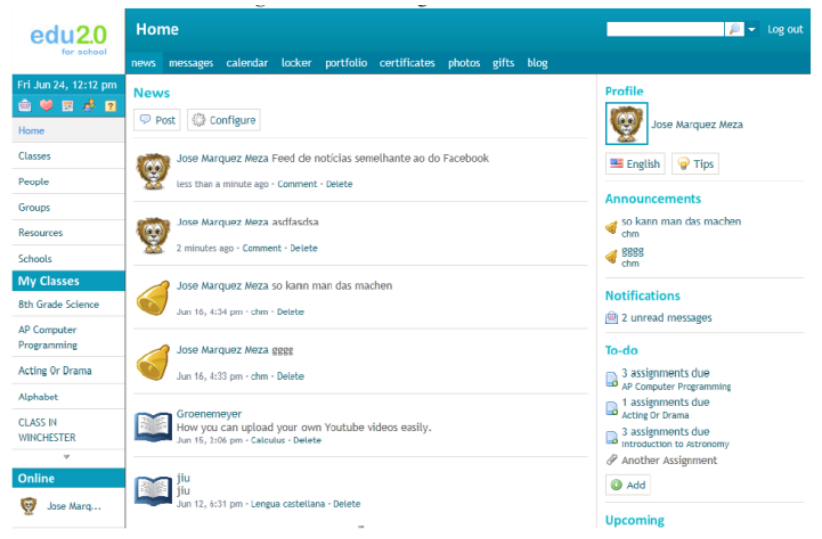

Figura 5 - Interface gráfica do Edu 2.0. (Fonte [36]).

Os autores defendem que LMSs baseados nas tecnologias da Web 2.0 (como redes sociais, wikis, blogs, folksonomias, fóruns) têm potencial para estimular a participação ativa e produção individual do conhecimento, permitir a realização de atividades de aprendizado formal e informal, extrair o melhor da inteligência coletiva e dar suporte ao processo de aprendizado dinâmico e aberto. Além disso, os autores declaram que o sistema faz parte de uma nova geração de LMSs, a qual eles denominam LMS 2.0. De acordo com seu web site oficial [36], o Edu 2.0 disponibiliza as seguintes funcionalidades: criação de classes e grupos; blogs, fóruns e calendários que podem ser pessoais, dos grupos e das classes; armário, um local reservado onde os usuários podem armazenar e visualizar recursos privados; álbum de fotos pessoal; bate-papo; wikis; questionários, enquetes, frequência dos alunos e caderno de notas; aplicação para dispositivos móveis - iPhone, iPad, Android e Blackberry; contas para os pais, com acesso às classes, notas e professores; integração com Facebook e Twitter. Além disso, o Edu 2.0 possui características de redes sociais, permitindo aos participantes construírem e visualizarem perfis, criarem listas de amigos, enviarem mensagens diretas uns aos outros, criação de grupos com interesses semelhantes e feed de notícias semelhante ao do Facebook [36]. De fato, toda interface gráfica do Edu 2.0 se assemelha à do Facebook, como mostrado na Figura 5. 


\section{TRABALHO PROPOSTO}

Esta seção apresenta os detalhes do trabalho proposto, chamado de SocialMoodle, o qual consiste no desenvolvimento de uma arquitetura baseada em agentes de software para realizar a integração do LMS Moodle com diversos sites de redes sociais. O objetivo é agregar os SNS ao processo de ensino para utilizá-los como plataforma de comunicação entre alunos e professores e para explorar o seu potencial de atrair e engajar os estudantes. A partir dessa arquitetura, as informações geradas no ambiente Moodle podem ser automaticamente replicadas em diferentes SNS e, por outro lado, os estudantes podem utilizar os sites de redes sociais para interagir com os recursos do Moodle. Neste trabalho, a arquitetura proposta foi implementada de forma a integrar o Moodle aos SNS Facebook e Twitter, respeitando as limitações de cada site. Entretanto, a arquitetura é flexível a ponto de possibilitar a inclusão de demais SNS.

\subsection{Funcionalidades}

As funcionalidades providas são:

- Vínculo - permite que os usuários do Moodle associem suas contas a um ou mais SNSs;

- Forúns - quando novos fóruns, discussões ou posts são criados no Moodle, os alunos interessados nesse evento são notificados através dos SNS.

- Enquetes - de forma semelhante aos fóruns, os alunos recebem mensagens informando quando novas enquetes são criadas no Moodle;

- Questionário - além de enviar mensagens aos estudantes quando novos questionários são criados, há também a possibilidade deles responderem aos questionários a partir do próprio SNS em uma interface bastante semelhante à do Moodle1. Essa é uma forma de fazer com que os alunos ganhem ainda mais familiaridade com o Moodle (Esta funcionalidade só é possível se o SNS escolhido oferecer às suas aplicações uma interface gráfica. No caso dos SNS escolhidos, essa funcionalidade pode ser implementada no Facebook mas não no Twitter.);

- Calendário - integra os calendários pessoais ou dos cursos aos calendários existentes nos SNS para manter os alunos bem informados a respeito dos eventos que acontecerão no curso. Além disso, notificações curtas são enviadas aos estudantes quando os eventos estiverem próximos de ocorrer;
- Notas - os estudantes recebem mensagens privadas informando suas notas à medida que os professores as publicam;

- Mensagens - integra o módulo de mensagens do Moodle, de forma que as mensagens enviadas através do LMS possam ser recebidas pelos estudantes nos sites de redes sociais, aumentando as chances de que estes, de fato, leiam-nas;

- Blogs - quando novos blogs forem criados, os sites de redes sociais são utilizados para informar os alunos e motivá-los a voltarem ao LMS para lerem os novos posts;

- Wikis - de maneira semelhante aos blogs, os alunos são notificados nos SNSs quando forem criadas novas páginas nos wikis do curso, estimulando-os a contribuírem para que o conteúdo gerado se torne cada vez mais completo.

\subsection{Arquitetura}

A arquitetura proposta consiste dos seguintes componentes fundamentais: (i) Agente de Configuração: responsável por inicializar o ambiente de execução, isto é, instanciar os demais agentes de software; (ii) Agentes de Módulo: para cada módulo do Moodle alvo da integração (e.g. Fórum, Questionário), há um agente desse tipo. Esses agentes têm a função de monitorar a base de dados do moodle e identificar quando houver novas informações que devam ser replicadas nos SNS (e.g. notificar a criação de um fórum); (iii) Ontologia do Moodle: descreve o domínio de aplicação do Moodle, sendo utilizada para definir uma linguagem básica comum a todos os agentes para proporcionar a comunicação e o entendimento entre eles; (iv) Agentes de Integração: responsáveis por encaminhar as informações identificadas pelos Agentes de Módulo para serem replicadas nos sites de redes sociais. Para cada SNS com o qual se deseja integrar o Moodle, deve-se especificar um agente deste tipo, o qual possui conhecimento a cerca das características desse SNS e de como cada informação deverá ser publicada; (v) Ontologia do SNS: descreve os conceitos relativos a um SNS específico. Representa o conhecimento que o Agente de Integração possui a respeito desse site de redes sociais; (vi) XML de Integração: utilizado pelo Agente de Integração, esse arquivo XML define de maneira padronizada como cada informação gerada no Moodle deverá ser apresentada no SNS; (vii) Aplicação Web: utiliza diretamente a API do SNS para publicar as informações, e ainda oferece uma interface gráfica por meio da qual os estudantes podem utilizar os recursos do Moodle. A Figura 6 apresenta a arquitetura do mecanismo proposto. Nas subseções seguintes são descritos cada um de seus componentes. 


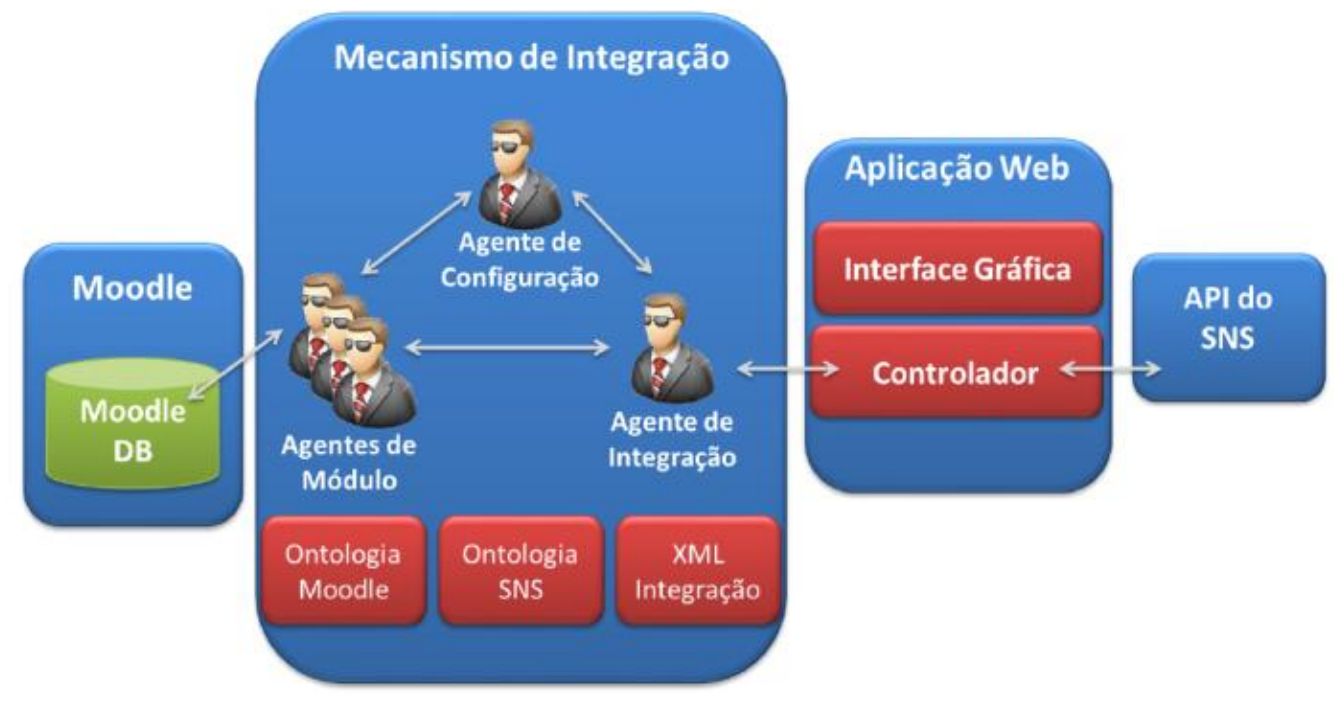

Figura 6 - Arquitetura do mecanismo proposto.

\subsection{Ontologia do Moodle}

A Ontologia do Moodle descreve o domínio de aplicação do LMS Moodle. Essa ontologia é utilizada para dotar os Agentes de Módulo do conhecimento necessário para realizar as suas atribuições. Além disso, essa ontologia é utilizada para definir uma linguagem básica comum a todos os agentes para proporcionar a comunicação e o entendimento entre eles. De forma geral, cada módulo do Moodle possui uma tabela (ou um conjunto de tabelas) no banco de dados que contém todas as informações relativas a ele. Os agentes de software monitoram essas tabelas afim de determinar quando ocorrem atualizações nesses módulos. Esta ontologia define os conceitos necessários para que os agentes compreendam as informações contidas no banco de dados e possam se comunicar com os demais agentes da aplicação. Portanto, para cada módulo integrado (e.g. Fórum, Questionário), existe um conceito correspondente nessa ontologia. A Tabela 1 apresenta uma partição dessa ontologia, na qual é descrito o domínio do módulo de mensagens.

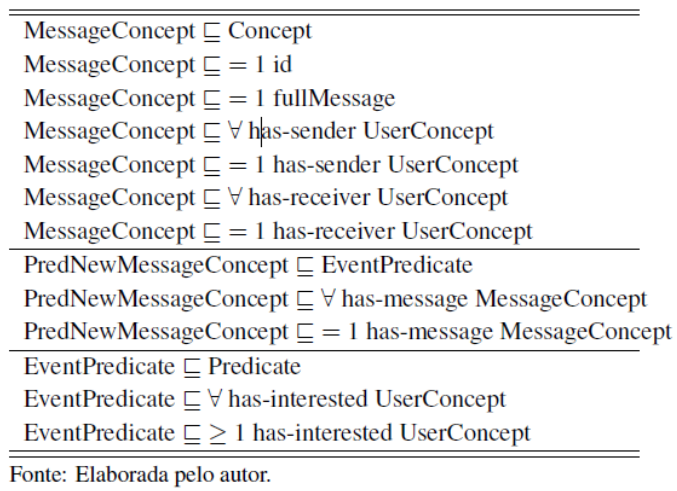


configurar a conexão JDBC com o banco de dados do Moodle. $\mathrm{O}$ arquivo agentsConfig.xml, especifica quais os demais agentes que serão instanciados na aplicação. Nesse arquivo, cada agente é definido entre as tags $<$ agent $>$, onde define-se o nome do agente que será criado, a classe Java que implementa esse agente e parâmetros opcionais que serão passados ao construtor do agente. Tendo em vista que os Agentes de Módulo possuem comportamentos bastante semelhantes, foi criado um agente genérico, implementado na classe MoodleAgent.java, o qual possui o comportamento mais comum entre os agentes desse tipo. Esse agente recebe em seu construtor uma String que representa o nome da propriedade de interesse desse agente. O comportamento desse agente genérico é o esperado para boa parte dos módulos, como Fóruns ou Enquetes, porém em alguns casos só parte desse comportamento se aplica corretamente. Por exemplo, para o módulo de Mensagens, o monitoramento da tabela e a instanciação do conceito estão corretos, porém os interessados no evento (de uma nova mensagem sendo enviada)

não são todos os participantes daquele curso, mas somente o destinatário da mensagem. Pensando nisso, foram definidos alguns pontos de extensão na classe MoodleAgent, de forma que é possível personalizar algumas ações, como é o caso do agente responsável pelo módulo de mensagens, mas ainda reaproveitar parte do comportamento definido no agente genérico. A Figura 7 apresenta um diagrama de classes mostrando todas as especializações da classe MoodleAgent que foram criadas para dar suporte aos comportamentos que são um pouco diferentes do agente genérico. Além disso, caso seja necessário, é possível utilizar um Agente de Módulo com uma implementação completamente personalizada, como é o caso do agente responsável pela autenticação dos usuários. Para isso, basta definir corretamente no arquivo agentsConfig.xml a classe que se deseja utilizar para esse agente.

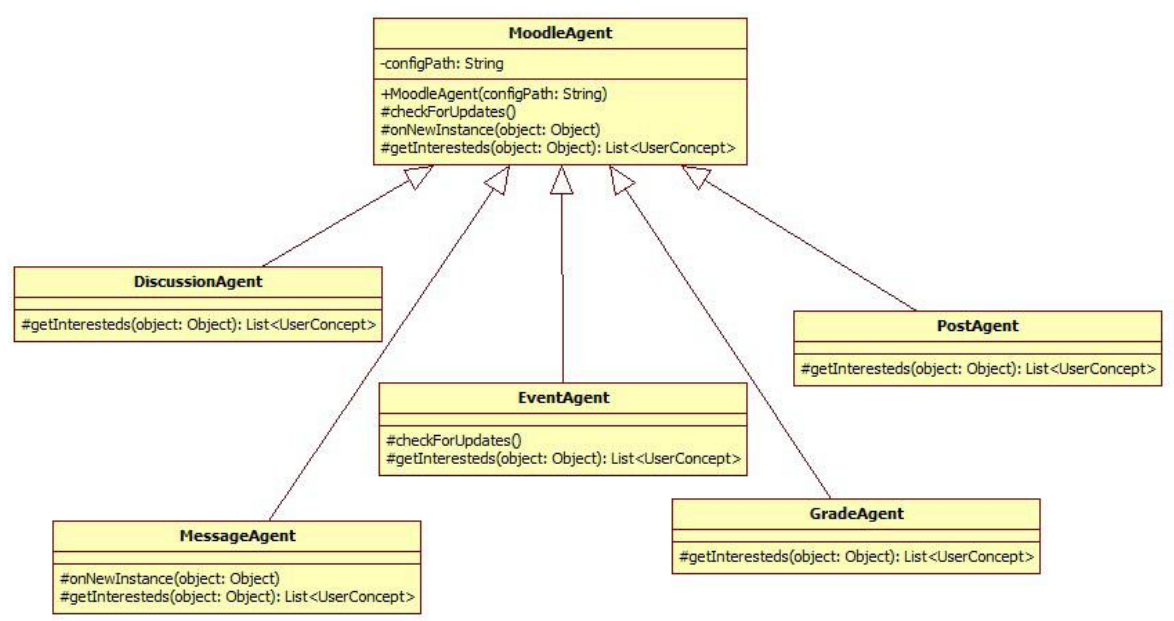

Figura 7: A classe MoodleAgent e suas especializações.

\subsection{Agente de Integração}

O Agente de Integração pode ser entendido como uma ponte que interliga o Moodle ao SNS desejado. Este agente possui duas funções. Por um lado, ele recebe mensagens dos Agentes de Módulo notificando sobre eventos que ocorreram no Moodle e em seguida replica a informação recebida de forma apropriada no site de redes sociais utilizado. Por outro lado, ele recebe o resultado das interações dos usuários com o SNS e as encaminha para o Agente de Módulo apropriado.Este agente utiliza um conjunto de regras para decidir como proceder mediante cada mensagem recebida. Essas regras são especificadas em um arquivo XML qualquer, cuja localização é passada para o construtor desse agente. Para cada SNS integrado, deve existir uma instância do Agente de Integração utilizando uma ontologia e um conjunto de regras específico para esse site.

\subsection{Aplicação do SNS}

A Aplicação do SNS é o componente arquitetural que de fato publica as informações no site de redes sociais. Para cada SNS integrado ao Moodle, deve existir uma aplicação Web que utiliza a API provida pelo site para interagir com ele. Esta aplicação oferece um conjunto de Web Services, por meio dos quais o Agente de Integração executa as ações definidas em suas regras. Dependendo das características do SNS utilizado, essa aplicação pode ou não oferecer uma interface gráfica aos usuários. Quando isso ocorre, essa aplicação pode oferecer aos usuários a possibilidade de interagir com os recursos do Moodle a partir dessa interface, isto é, sem sair do site de redes sociais. Nesse caso, a aplicação deve ainda prover um agente de software, o qual vai se comunicar com o Agente de Integração para encaminhar o 
resultado das interações dos usuários desde o SNS até o Moodle.

\section{AVALIAÇÃO DA ARQUITETU- RA PROPOSTA}

Esta seção tem o propósito de demonstrar a viabilidade da arquitetura proposta através da sua implementação para realizar a integração do Moodle com: 1) Twitter; 2) Facebook.

\subsection{Integração Moodle - Twitter}

O objetivo dessa Seção é demonstrar como a arquitetura proposta foi utilizada para integrar o LMS Moodle ao Twitter. A ideia dessa integração é utilizar o SNS como plataforma para manter os estudantes informados do que ocorre nos cursos. Nesse sentido, optou-se por utilizar o recurso do Twitter de mensagens diretas. Esse tipo de mensagem é enviada a um usuário em particular, de forma que somente ele pode visualizá-la. Assim, sempre que um novo recurso é criado no Moodle, os usuários interessados nesse evento recebem uma mensagem direta em seus perfis do Twitter. Essa opção foi escolhida por manter a privacidade dos estudantes, visto que não seria interessante que algumas das informações enviadas ao SNS fossem de conhecimento público, como por exemplo as notas dos alunos. Os seguintes módulos do Moodle foram escolhidos como alvo da integração, isto é, os estudantes são notificados quando houver atualizações em qualquer um desses módulos: Questionário, Enquete, Bate-papo, Fórum, Mensagem, Notas, Calendário, Blogs e Wikis. Além desses módulos, também foi necessário criar uma estrutura para permitir que os usuários associassem suas contas do Twitter às contas do Moodle. Para tal, foi criado um plugin na forma de um bloco no Moodle, por meio do qual os usuários autenticados no LMS podem conceder à aplicação as permissões necessárias para enviar-lhes as mensagens desejadas. O plugin foi implementado em PHP, utilizando-se a biblioteca TwitterOAuth para o acesso à API do Twitter. $\mathrm{O}$ próximo passo na implementação foi definir a Ontologia do Twitter. De acordo com a arquitetura especificada, essa ontologia estende a Ontologia do Moodle, herdando seus conceitos e relacionamentos. Dessa forma, a ontologia resultante é bastante simples, tendo sido necessário definir apenas o conceito de mensagem direta. A Tabela 2 apresenta essa ontologia.

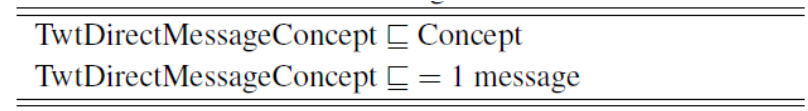

Fonte: Elaborada pelo autor.

Tabela 2: Ontologia do Twitter.

Uma vez que a ontologia foi definida, foi necessário implementar a Aplicação do SNS, isto é, a aplicação Web que utiliza a API do Twitter para enviar as mensagens. Como a integração entre o Moodle e o Twitter ocorre apenas através do envio de mensagens diretas, foi necessária a implementação apenas de um Web Service com essa função. Essa aplicação foi implementada em Java utilizando-se as bibliotecas jersey, para criar o Web Service, e Twitter4J para utilizar a API do Twitter e enviar as mensagens diretas aos usuários. $\mathrm{O}$ último passo necessário para conseguir a integração foi definir o conjunto de regras por meio das quais o Agente de Integração decide a ação a ser tomada quando um evento acontece. Como resultado da integração, os usuários recebem as mensagens diretas em seus perfis do Twitter sempre que um novo recurso é criado no Moodle. 


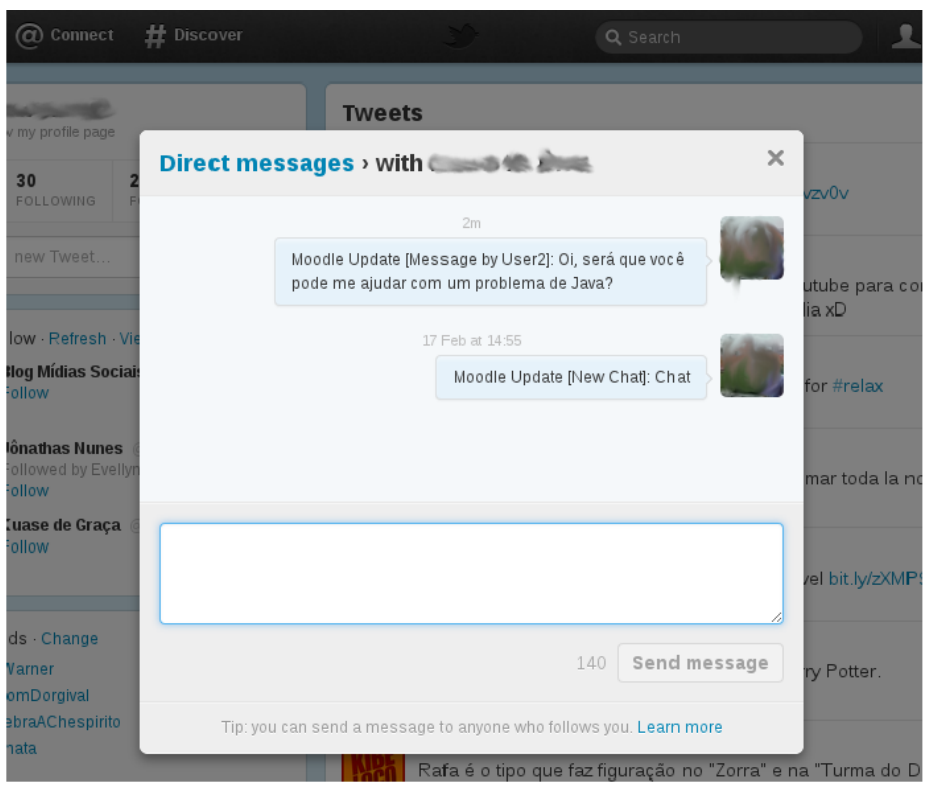

Figura 7: Resultado da integração do Moodle com o Twitter.

A Figura 7 demonstra as mensagens que um usuário recebeu notificando-o que um novo bate-papo foi criado e que um outro usuário o envio de uma mensagem pelo Moodle.

\subsection{Integração com o Facebook}

Esta Seção apresenta como a arquitetura proposta foi utilizada para integrar o Moodle ao SNS Facebook. A integração do Moodle com o Facebook implementa três diferentes funcionalidades: i)visto que esse SNS oferece uma função de calendário, os eventos definidos nos calendários do Moodle são replicados no calendário do Facebook; ii) para os demais módulos, sempre que há alguma atualização, os estudantes recebem publicações em seus murais; iii) para o módulo de questionários, foi implementada uma funcionalidade adicional, que permite que os alunos respondam a um determinado questionário a partir do próprio SNS. Essa funcionalidade é possível porque o Facebook oferece uma interface gráfica às suas aplicações. O primeiro passo para implementar essa integração foi definir a Ontologia do Facebook, por meio da qual o Agente de Integração compreende os conceitos relativos a esse SNS. A Tabela 3 apresenta essa ontologia. É importante destacar que essa ontologia estende a Ontologia do Moodle.

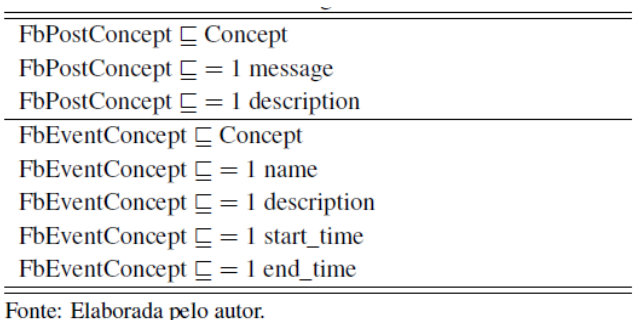

Tabela 3: Ontologia do Facebook.

Após a definição da ontologia, foi implementada a aplicação Web por meio do qual os estudantes podem: associar as suas credencias do Facebook às suas contas do Moodle; receber as publicações nos murais; sincronizar o calendário com o do Moodle; responder aos questionários. Essa aplicação foi desenvolvida em Java, fazendo uso das seguintes bibliotecas: Jersey, para definir os Web Services; HttpClient, para utilizar a API do Facebook por meio de requisições HTTP; e Struts2, para especificar a interface Web. A partir dessa aplicação, os usuários, no ambiente do Facebook, podem dar as permissões que a aplicação necessita e em seguida podem inserir as credencias do seu usuário Moodle, efetuando portanto o vínculo entre os dois sistemas. A Figura 8 apresenta a tela onde o usuário autoriza a aplicação. 


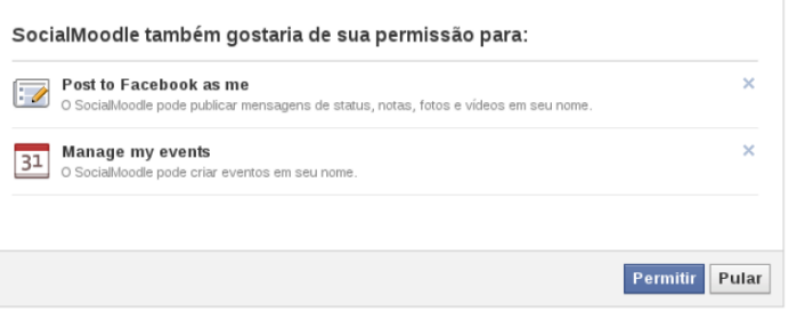

Figura 8: Tela do Facebook onde o usuário autoriza o uso da aplicação como encaminhar as informações do Moodle ao SNS e vice-versa. Como resultado da integração, os estudantes recebem publicações nos seus murais, podem responder aos questionários do Moodle a partir do Facebook e seus calendários são sincronizados. A Figura 9 mostra, respectivamente, uma publicação no mural de um estudante e a tela para responder aos questionários.

Além da aplicação Web, foi necessário especificar as regras por meio das quais o Agente de Integração define

Publicação no mural do estudante notificando a criação de um novo questionário.

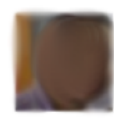

Moodle Update [New Quiz]: Final Test

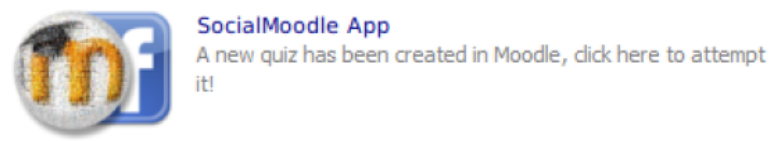

Tela para responder aos questionários do Moodle.

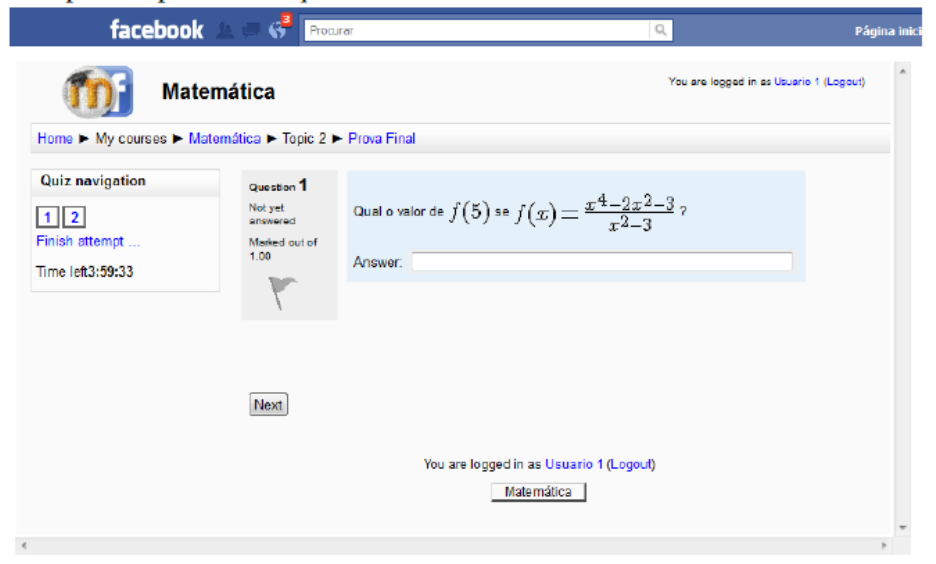

Figura 9: Publicação e Questionário

Além da aplicação Web, foi necessário especificar as regras por meio das quais o Agente de Integração define como encaminhar as informações do Moodle ao SNS e vice-versa. O Código, Figura 10, apresenta uma partição do arquivo fbIntegration.xml, o qual define essas regras.

\section{COMPARAÇÃO COM TRABA- LHOS RELACIONADOS}

A Figura 11 apresenta uma tabela comparando este trabalho, denominado SocialMoodle, com os demais trabalhos relacionados. Os critérios escolhidos para comparação foram: vínculo, possibilitar que os usuários associem as credenciais do Moodle às dos SNSs; autenticação, permitir realizar o login no Moodle utilizando as credenciais do SNS associado; módulos do Moodle, rea- lizar a integração dos módulos já existentes no Moodle; LMS -> SNS, conduzir as informações do Moodle para os sites de redes sociais; SNS -> LMS, encaminhar as informações dos SNSs para o Moodle; e flexibilidade, oferecer suporte à integração com diferentes sites de redes sociais. Este trabalho se diferencia dos demais principalmente por possibilitar o fluxo de informações do Moodle para os SNSs, de forma que os alunos podem estudar utilizando uma ferramenta que lhes é bastante 


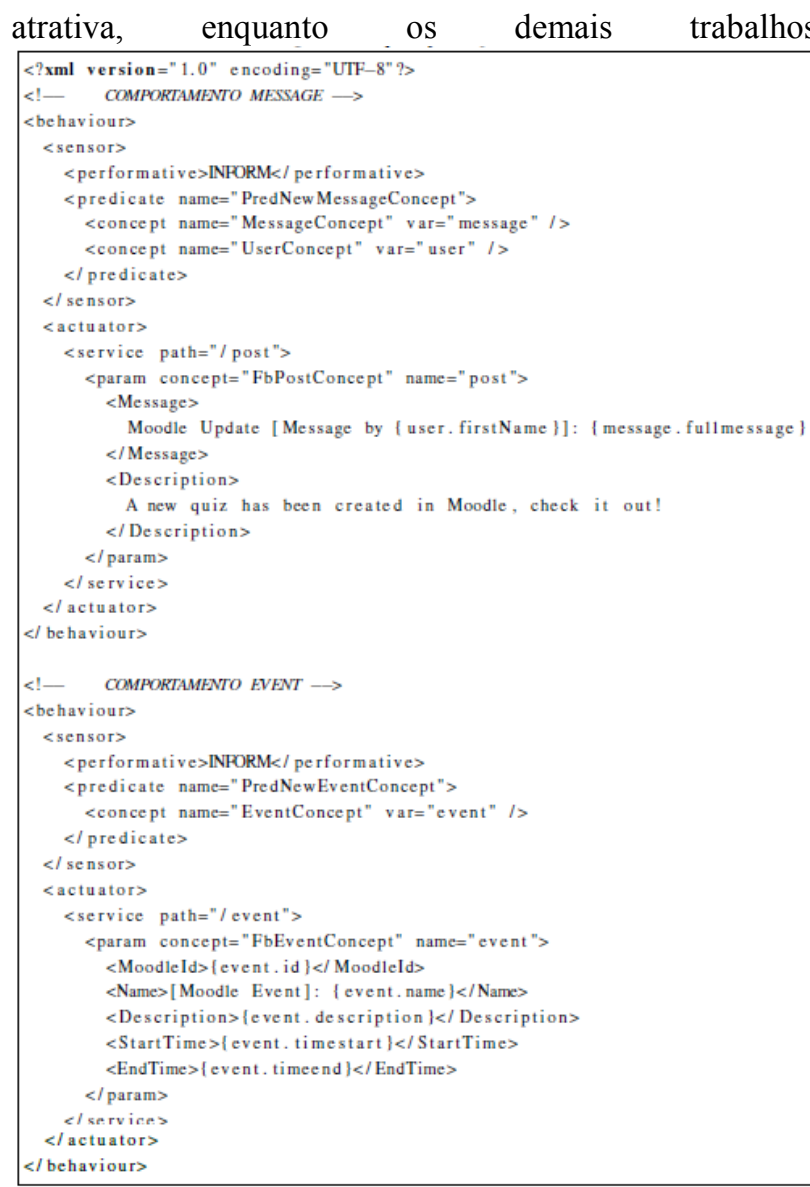

Figura 10: Script de Integração do Agente.

focam apenas no caminho inverso, isto é, dos SNSs para o Moodle; e por oferecer flexibilidade na escolha dos sites de redes sociais que serão integrados. Enquanto os outros trabalhos oferecem uma quantia fixa (normalmente Facebook e/ou Twitter) de SNSs com os quais se pode integrar o Moodle, este trabalho apresenta uma arquitetura com diversos pontos de extensão que podem ser explorados para dar suporte a qualquer site de redes sociais.

\begin{tabular}{|l|c|c|c|c|}
\hline CRITÉRIOS & Fulton(2010) & (INTERACTYX, 2011 & EDU2.0, 2011 & SocialMoodle \\
\hline Vinculo & $\mathrm{x}$ & $\mathrm{x}$ & $\mathrm{x}$ & $\mathrm{x}$ \\
\hline Autenticação & $\mathrm{x}$ & $\mathrm{x}$ & $\mathrm{x}$ & \\
\hline $\begin{array}{l}\text { Módulos do } \\
\text { Moodle }\end{array}$ & & & & $\mathrm{x}$ \\
\hline LMS -> SNS & & & parcialmente & $\mathrm{x}$ \\
\hline SNS -> LMS & $\mathrm{x}$ & $\mathrm{x}$ & $\mathrm{x}$ & $\mathrm{x}$ \\
\hline Flexibilidade & & & & $\mathrm{x}$ \\
\hline
\end{tabular}

Figura 11: Comparação com trabalhos relacionados

\section{CONSIDERAÇÕES FINAIS}

O objetivo deste trabalho foi apresentar uma arquitetura baseada em agentes de software e ontologias para promover a integração do sistema de gestão de aprendizagem Moodle a diferentes sites de redes sociais com o intuito de aproveitar o entusiasmo dos alunos por esses sites em favor da educação. A arquitetura apresentada pode ser usada para conduzir informações criadas no Moodle aos sites de redes sociais para, por exemplo, manter os estudantes atualizados do que ocorre nos cursos. A partir dessa arquitetura também é possível replicar informações geradas nos SNS de volta ao Moodle como, por exemplo, permitir que os estudantes interajam com os recursos do Moodle a partir dos sites de redes sociais. Além disso, a arquitetura é flexível a ponto de permitir o uso de diversos SNS ao mesmo tempo. Para demonstrar a viabilidade da proposta foi realizado um estudo de caso, no qual a arquitetura proposta foi implementada para integrar o Moodle aos SNS Facebook e Twitter. Diante disso, é possível afirmar que os objetivos desse trabalho foram alcançados. Como trabalho futuro é necessário implementar e avaliar a proposta desse trabalho no contexto de um curso com usuários reais, afim de identificar o impacto que o uso dessa integração exerce sobre o processo de aprendizagem. Os resultados dessa pesquisa foram bem aceitos pela comunidade científica da área de informática na educação. Este trabalho defende a ideia de que os sites de redes sociais tem um grande potencial, ainda pouco explorado, para auxiliar e efetivamente melhorar a educação. A arquitetura apresentada objetiva fazer uso desse potencial, trazendo benefícios para alunos e professores.

\section{Referências}

[1] C. Lemke, E. Coughlin, L. Garcia, D. Reifsneider, J. Baas. Leadership for Web 2.0 in Education: Promise \& Reality. CoSN, Culver City, 2009.

[2] J. Ahn. The effect of social network sites on adolescents' social and academic development: Current theories and controversies. J. Am. Soc. Inf. Sci.1435-1444, 2011.

[3] M. Ito, S. Baumer, M. Bittanti et al. Hanging Out, Messing Around and Geeking Out. MIT Press, Cambridge, 2009.

[4] P. Ractham, D. Firpo. Using Social Networking Technology to Enhance Learning in Higher Education: A Case Study using Facebook. In Proceedings of the 44th Hawaii International Conference on System Sciences. páginas 1-10, 
2011.

[5] H. Jiang, M. Tang. Web-Based Learning Platforms Integrating Social Networking for Design Education at High Schools in China. In International Conference on Computational Intelligence and Software Engineering (CiSE). páginas 1-3, 2010 .

[6] H. Hodgins. Into the Future A Vision Paper. Commission on Technology and Adult Learning, 2007.

[7] D. McInerney, V. McInerney. Educational Psychology Constructing Learning. Prentice Hall, Austrália, 2002.

[8] M. Molina, D. Sales. Knowledge Transfer and Information Skills for Student-Centered Learning in Spain. Portal: Libraries and the Academy, 8(1):53-74, 2008.

[9] M. Chatti, S. Srirama, D. Kensche, Y. Cao. Mobile Web Services for Collaborative Learning. In Fourth IEEE International Workshop on Wireless, Mobile and Ubiquitous Technology in Education. páginas 129-133, 2006.

[10] E. Clark. When Researchers Swim Upstream: Reflections on an Unpopular Argument about Learning from Media. Educ. Technol, 31:34-40, 1991.

[11] E. Clark. Reconsidering Research on Learning from Media. Review of Educational Research, 53(4):445-459, 1983.

[12] R. Rivera. Instruction over Online Social Networks: Where does the Platform Lead?. In International Conference on Technology for Education (T4E). páginas 209-215, 2010.

[13] S. Wasserman, K. Faust. Social Network Analysis: Methods and Applications (Structural Analysis in the Social Sciences. Cambridge University Press, Cambridge, 1994.

[14] L. Garton, C. Haythornthwaite, B. Wellman. Studying Online Social Networks. Journal of Computer-Mediated Communication, 1997.

[15] O. Serrat. Social Network Analysis. Knowledge Solutions. 1-3, 2009.

[16] N. Gonzales. Check Facebook. http://www.checkfacebook.com/. Set, 2012.

[17] M. Anzures-Garcia, L. Sanchez-Galvez, E. Lopez-Melendez et al. Platform to supports learning based on Social Network, Web Intelligence and CSCL. In 20th International Conference on Electronics, Communications and Computer. páginas 201-205, 2010.

[18] D. Boyd, N. Ellison. Social Network Sites: Definition, History, and Scholarship. Journal of Computer-Mediated Communication, 13(1):210230, 2007.

[19] J. Breslin, S. Decker. The Future of Social Networks on the Internet: The Need for Semantics. IEEE Internet Computing, 11(6):86-90, 2007.

[20] L. Lockyer, J. Patterson. Integrating Social Networking Technologies in Education: a Case Study of a Formal Learning Environment. In Eighth IEEE International Conference on Advanced Learning Technologies. páginas 529-533, 2008.

[21] M. Li, Z. Liu. The Role of Online Social Networks in Students' E-Learning Experiences. In International Conference on Computational Intelligence and Software Engineering, páginas 1-4, 2009.

[22] S. Maglajlic, D. Helic, C. Trattner. Social Networks and eLearning: New Model for Learning at Workplace. In 32nd International Conference on Information Technology Interfaces, páginas 373-378, 2010.

[23] J. E. Cain. Online Social Networking Issues Within Academia and Pharmacy Education. Am J Pharm Educ, 2008.

[24] T. R. Gruber. A Translation Approach to Portable Ontology Specifications. Knowledge Acquisition, 5(2):199-220, 1993.

[25] W3C. OWL Web Ontology Language Overview. http://www.w3.org/TR/owl-features/. Set, 2012.

[26] F. Bellifemine, G. Caire, D. Greenwood. Developing Multi-Agent Systems with JADE. Wiley, 2007.

[27] S. Russell, P. Norvig. Artificial Intelligence: A Modern Approach. Pearson Education, 2003.

[28] M. Wooldridge. An Introduction to MultiAgent Systems. John Wiley \& Sons, 2002.

[29] C. Shiang, N. Kulathuramaiyer, S. Loke. Software Agent Negotiation Development: An Experience Report. In ISDA '06: Sixth International Conference on Intelligent Systems Design and Applications. páginas 881-886, 2006.

[30] FIPA. The Foundation for Intelligent Physical Agents. http://www.fipa.org/. Set, 2012.

[31] V. Kelemen. Simple Example for using the JadeGateway class. 
http://jade.tilab.com/doc/tutorials/JadeGateway.p df. Set, 2012.

[32] A. Fulton. Facebook Connect. http://docs.moodle.org/20/en/Facebook \packag e. Jun, 2011.

[33] W. Ho. Integrating Social Network Service into Learning Management System. In e-Learning Week Conference. 2010.

[34] Interactyx. Social Learning Management System (Social LMS) eLearning Software Solution. http://www.interactyx.com/request-elearningsoftware-demonstration. Jun, 2011.

[35] M. Ivanova, A. Popova. An Exploration of Formal and Informal Learning Flows in LMS 2.0: Case Study Edu 2.0. In IEEE/WIC/ACM International Joint Conferences on Web Intelligence and Intelligent Agent Technologies. páginas 227230, 2009.

[36] Edu2.0. EDU 2.0 for School: The Free, Easy Way to Teach and Learn Online. http://www.edu20.org/tour/features. Jun, 2011. 